\title{
Análise das Redes de Colaboração Científica no Simpósio Brasileiro de Sistemas Colaborativos
}

\author{
Beatriz Saburido Racca ${ }^{1}$, Juliana Baptista dos Santos França ${ }^{1,2,3}$, Bruna Diirr ${ }^{4}$ e \\ Vaninha Vieira dos Santos ${ }^{5}$
}

${ }^{1}$ Departamento de Computação (DECOMP) - Universidade Federal Rural do Rio de Janeiro (UFRRJ) - Seropédica, RJ, Brasil

${ }^{2}$ Programa de Pós-Graduação em Modelagem Matemática e Computacional (PPGMMC) - Universidade Federal Rural do Rio de Janeiro (UFRRJ) - Seropédica, RJ, Brasil
${ }^{3}$ Programa de Pós-Graduação em Gestão e Estratégia (PPGE) - Universidade Federal Rural do Rio de Janeiro (UFRRJ) - Seropédica, RJ, Brasil
${ }^{4}$ Programa de Pós-Graduação em Informática -Universidade Federal do Estado do Rio de Janeiro (UNIRIO) - Rio de Janeiro, RJ, Brasil
${ }^{5}$ Instituto de Matemática e Estatística -Universidade Federal da Bahia - Salvador, BA, Brasil

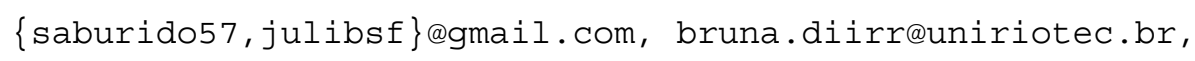

\begin{abstract}
The Brazilian Symposium on Collaborative Systems completes 16 editions in 2021, being the main CSCW event in Brazil. After a restructuring in 2019, due to a decline in participants at the event, a study of its recent characteristics is necessary. This paper proposes a retrospective study of the co-authorship networks from 2013 to 2019, in addition to other significant community analysis such as: the investigated research topics, the number of published articles, the active authors and institutions, the participants' geographic distribution and the gender distribution of published research.
\end{abstract}

Resumo. O SBSC completa 16 edições no ano de 2021, sendo o principal evento da área de CSCW no Brasil. Após uma reestruturação em 2019, por conta de um declínio de participantes no evento, se faz necessário um estudo de suas características recentes. Este artigo traz um retrospecto das redes de coautoria no período de 2013 a 2019, além de outras análises significativas sobre a comunidade como a quantidade de artigos publicados, os autores $e$ instituições ativas, a distribuição geográfica dos participantes e a distribuição por gênero das pesquisas publicadas.

\section{Introdução}

O Simpósio Brasileiro de Sistemas Colaborativos (SBSC) é o principal evento da área de CSCW no Brasil. Desde 2003, reúne anualmente pesquisadores, estudantes e profissionais do Brasil e exterior para apresentação e discussão dos últimos avanços da área [CESC 2020]. Após passar por alguns problemas, entre anos de 2010 e 2017, e um um hiato em 2018 [CESC 2017], o SBSC retomou suas atividades em 2019, contando com renovação de ideias, formatos de submissão e dinâmicas de apresentação, mudando completamente a estrutura do evento e visando sua expansão [SBSC 2019]. 
Nesse contexto, nota-se a importância de conhecer e reconhecer a comunidade que ativamente participa e colabora com o SBSC. Isso possibilita perceber fatores que possam reduzir a articulação da comunidade, bem como apoiar a reflexão sobre estratégias para ampliar parcerias e redes de colaboração. Redes de colaboração científicas são redes sociais formadas através da cooperação entre dois ou mais pesquisadores [Steinmacher et. al 2013, Gasparini et. al 2014, Dias et. al 2014], permitindo assim revelar diversos aspectos a respeito das comunidades científicas.

Este artigo visa investigar as redes de colaboração científica formadas por pesquisadores atuantes no SBSC, destacando: o estudo retrospectivo e análise da evolução do evento, bem como a rede de colaboração de coautoria dos pesquisadores estabelecidas em torno do simpósio. Esse estudo inspira-se em trabalhos anteriores com propósito similar [Steinmacher et al. 2013, Dias et al. 2014] e busca atualizar a análise e discussão das redes formadas nas edições mais recentes do SBSC, ocorridas entre 2013 e 2019. Steinmacher et al. (2013) analisaram uma rede de coautoria baseada na literatura publicada no SBSC até 2012, focando em tópicos de pesquisa e redes de colaboração estabelecidas na comunidade. Os autores apontam que a rede crescia a cada ano e novos grupos surgiam após a realização de trabalhos junto a pesquisadores sêniores e líderes de grupos de pesquisa. Também mostram a ausência de pesquisadores da região Centro-Oeste. Já Dias et al. (2014) investigaram as características da rede do SBSC através da interação entre os atores nesta comunidade, utilizando métricas clássicas de análise de redes. Os autores apontam uma predominância de participação de instituições da região Sudeste do Brasil, seguidas das regiões Nordeste, Sul, Norte e Centro Oeste.

O objetivo desta pesquisa é avançar as análises da rede de colaboração científica do SBSC, tendo por fundamentos os trabalhos relacionados e as questões de investigação propostas na Seção 2. Para isso, todos os artigos publicados no evento entre 2013 e 2019 foram analisados, possibilitando identificar a evolução das redes de colaborações científicas, pesquisadores com maior número de publicações, participação por instituições/regiões do Brasil, participação por gênero, participação por edição e quantitativo de artigos pesquisados. Os resultados também foram comparados com os obtidos em Steinmacher et al. (2013). As análises apresentadas no artigo são relevantes para que a comunidade do SBSC possa refletir sobre suas características particulares e parcerias formadas. Além disso, tais resultados podem apoiar a identificação de fatores ou padrões que levaram à redução da participação da comunidade no evento.

O artigo está estruturado da seguinte forma: a Seção 2 introduz as questões de investigação e o método adotado na pesquisa; a Seção 3 descreve e analisa os resultados encontrados; e a Seção 4 traz as considerações finais e indicações de trabalhos futuros.

\section{Questões de Investigação e Metodologia de Pesquisa}

Com a finalidade de caracterizar a rede de colaboração formada entre os pesquisadores atuantes no SBSC de 2013 a 2019, esta pesquisa está estruturada considerando as seguintes questões de investigação:

QI1. Quantos artigos foram publicados por categoria?

QI2. Quais são os autores e instituições mais ativos no SBSC no período investigado? 
QI3. Como se caracteriza a distribuição geográfica dos pesquisadores do SBSC?

QI4. Qual a distribuição por gênero das pesquisas publicadas no SBSC?

QI5. Como as redes de colaboração científicas dos pesquisadores no SBSC evoluíram?

Para conduzir a pesquisa, a metodologia adotada seguiu três etapas: (i) Coletar metadados dos artigos publicados; (ii) Construir a Rede de Colaboração Científica; e (iii) Apresentar os resultados alcançados.

\subsection{Coletar Metadados dos Artigos}

Inicialmente, foi realizado um levantamento das edições que tinham documentação do evento disponível. Observou-se que os sites das edições de 2013 e 2015 estavam fora do ar, suas documentações não estavam disponíveis na plataforma SBC Open Lib (SOL) e as buscas no Google pelos seus anais não retornavam os resultados desejados. Com isso, partiu-se para a inspeção do Lattes, visando a identificação de autores que participaram de edições do SBSC.

As strings de busca utilizadas foram o nome completo do evento e sigla, em buscas simples separadas, filtrando a partir do ano de 2013 e checando apenas a seção referente a produções bibliográficas. Os resultados de pesquisa foram analisados e os autores participantes alocados manualmente em uma planilha Excel, resultando em 41 autores diferentes. Uma vez obtida uma parte dos títulos dos artigos através da mineração do Lattes, o passo seguinte foi realizar a busca para cada artigo.

- Edição de 2013: Busca manual na plataforma ACM Digital Library.

- Edição de 2014: Anais disponíveis no site do evento.

- Edição de 2015: Busca manual de parte dos títulos dos artigos no Google, que resultou na identificação de um post no Facebook listando todos os artigos aceitos. Porém, nem todos os arquivos dos artigos foram recuperados.

- Edições de 2016, 2017 e 2019: Artigos disponíveis na plataforma SOL.

Os metadados coletados foram o nome do autor, afiliação na instituição, colaborações entre pesquisadores e quantidade de artigos publicados no SBSC.

\subsection{Construir Rede de Colaboração Científica}

Para elaborar a rede de colaboração científica do SBSC no período de 2013 a 2019, foi necessário obter a lista de autores por artigo publicado no evento. Esta atividade foi realizada juntamente com a coleta dos títulos dos artigos. Isto é, assim que um título era coletado, os nomes e universidades eram coletados em conjunto.

Com os artigos devidamente catalogados, o próximo passo foi a extração das redes de colaboração científica. Para isso, os autores foram cadastrados manualmente em uma planilha Excel, junto com seu número de publicações (totais e por evento) e colaborações. Em seguida, padronizou-se os nomes dos autores nos diferentes artigos, pois alguns possuíam variações de escrita (casos com até quatro variações de nome). 
Após isso, criou-se um grafo, que permite a visualização das redes de colaboração científica formadas, utilizando o software yEd Graph Editor ${ }^{l}$. Os nós foram definidos automaticamente usando os nomes da planilha e as relações foram criadas manualmente.

\subsection{Apresentar Resultados Alcançados}

Os resultados alcançados estão alinhados com as cinco questões de investigação estabelecidas, sendo considerados os dados levantados: (i) nos artigos publicados; (ii) no currículo Lattes dos pesquisadores envolvidos no SBSC; e (iii) nas redes sociais do SBSC, considerado o período desta investigação. Detalhes sobre o levantamento desses dados e discussões sobre os resultados alcançados serão apresentados na Seção 3.

\section{Resultados Encontrados e Discussões}

Em relação ao quantitativo de artigos publicados por categoria, a quantidade de trabalhos completos publicados no SBSC variou consideravelmente. A edição de 2014 contou com o menor número de trabalhos finalizados, contando com 10 trabalhos, e a edição de 2016 contou com 25 trabalhos (Figura 1). A edição de 2019 recebeu trabalhos de diferentes vertentes (desenho de pesquisa, pesquisas em andamento e pesquisas finalizadas), publicados em dois anais: um principal e um estendido (Figura 2).

Sobre os autores e instituições mais ativas no SBSC entre 2013 e 2019, percebe-se que os autores que mais publicaram foram Carla Berkenbrock (UDESC - 12 artigos), Glívia Barbosa (CEFET-MG - 11 artigos) e Adriana Vivacqua (UFRJ - 10 artigos). Já em relação às instituições, notam-se três universidades com maior concentração de pesquisadores com publicações no SBSC: UFRJ (RJ), UFAM (AM) e UDESC (SC).

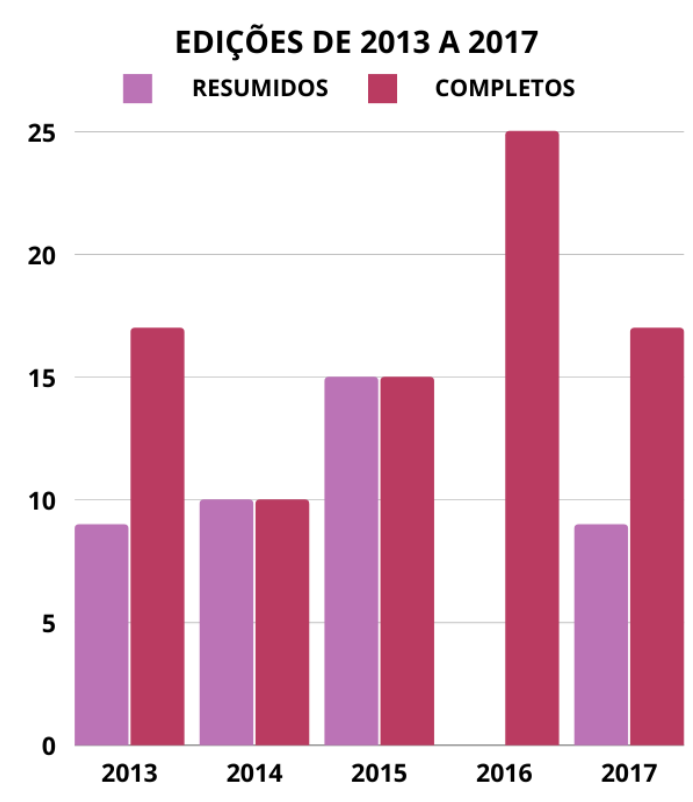

Figura 1. Categorias (2013 a 2017)

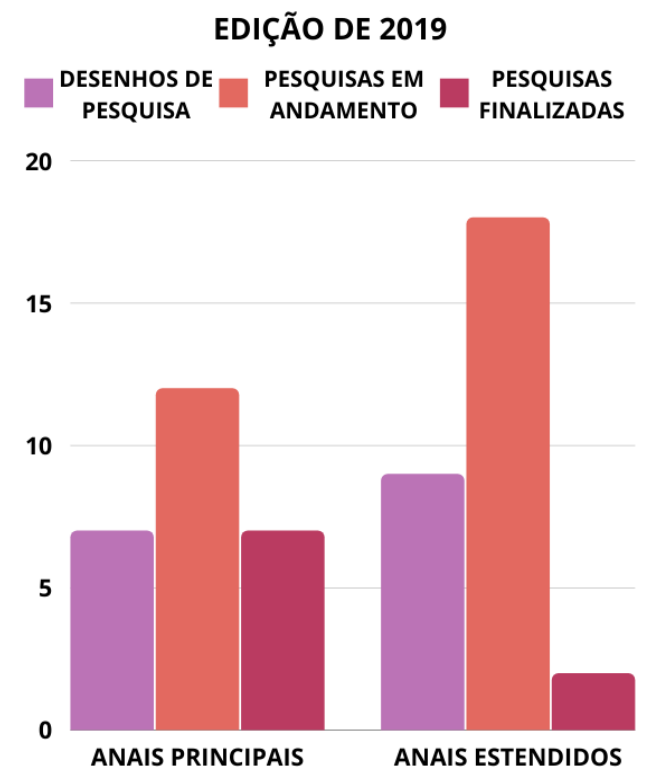

Figura 2. Categorias (2019)

\footnotetext{
${ }^{1}$ https://www.yworks.com/products/yed
} 
A respeito da distribuição geográfica dos pesquisadores, a maior parte dos autores são filiados a instituições do Sudeste, seguidos das regiões Sul, Norte, Nordeste e Centro Oeste, nessa ordem (Figura 3). Nota-se um aumento de autores das regiões Sul e Norte, talvez devido ao surgimento de novos núcleos de pesquisa nessas regiões e que podem ter contribuído para a introdução de novos autores na rede do SBSC. Também observa-se que apenas 8 dos 411 autores do SBSC são da região Centro-Oeste, o que reforça a sugestão de Steinmacher et. al (2013) sobre a necessidade de ações para fomentar o desenvolvimento da comunidade nessa área.

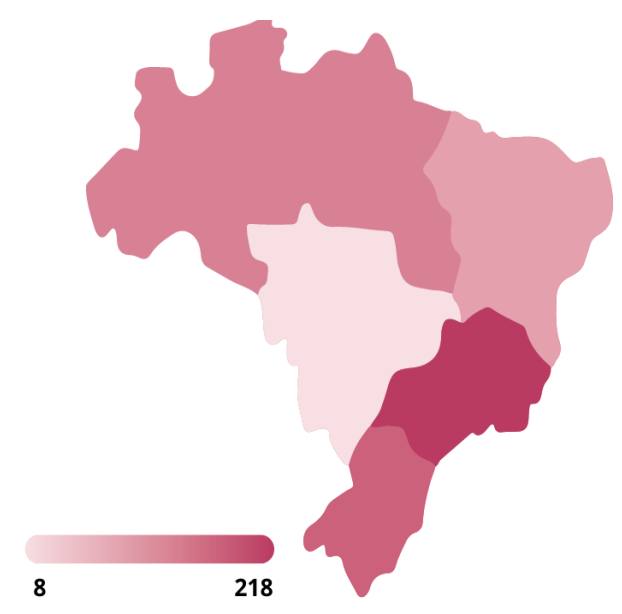

Figura 3. Pesquisadores pelo Brasil

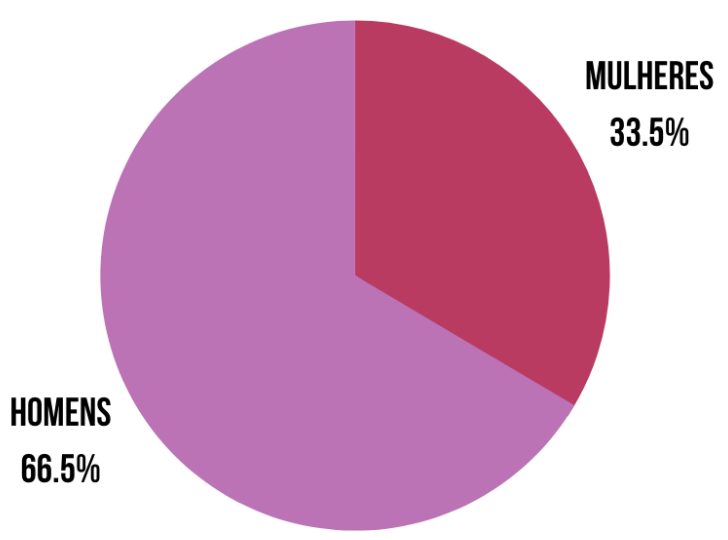

Figura 4. Distribuição por gênero

Quanto a distribuição por gênero das pesquisas publicadas, apesar dos 3 autores com mais de 10 publicações entre 2013 e 2019 serem mulheres, pessoas do sexo feminino compõem apenas 33,5\% dos autores que publicaram no SBSC (Figura 4). Relativo à evolução das redes de colaboração científicas no SBSC, a Figura 5 ilustra a rede das colaborações de coautoria de 411 pesquisadores. Os nós representam os autores e as arestas representam a coautoria em artigos. O diâmetro e cor dos nós representam a quantidade de artigos publicados por autor e apenas autores com 5 ou mais publicações foram rotulados, para evitar a poluição visual da imagem. A partir da visualização das interações apresentadas, observa-se que existem 3 autores isolados, ou seja, que não possuem nenhuma rede de colaboração com demais autores do SBSC. 


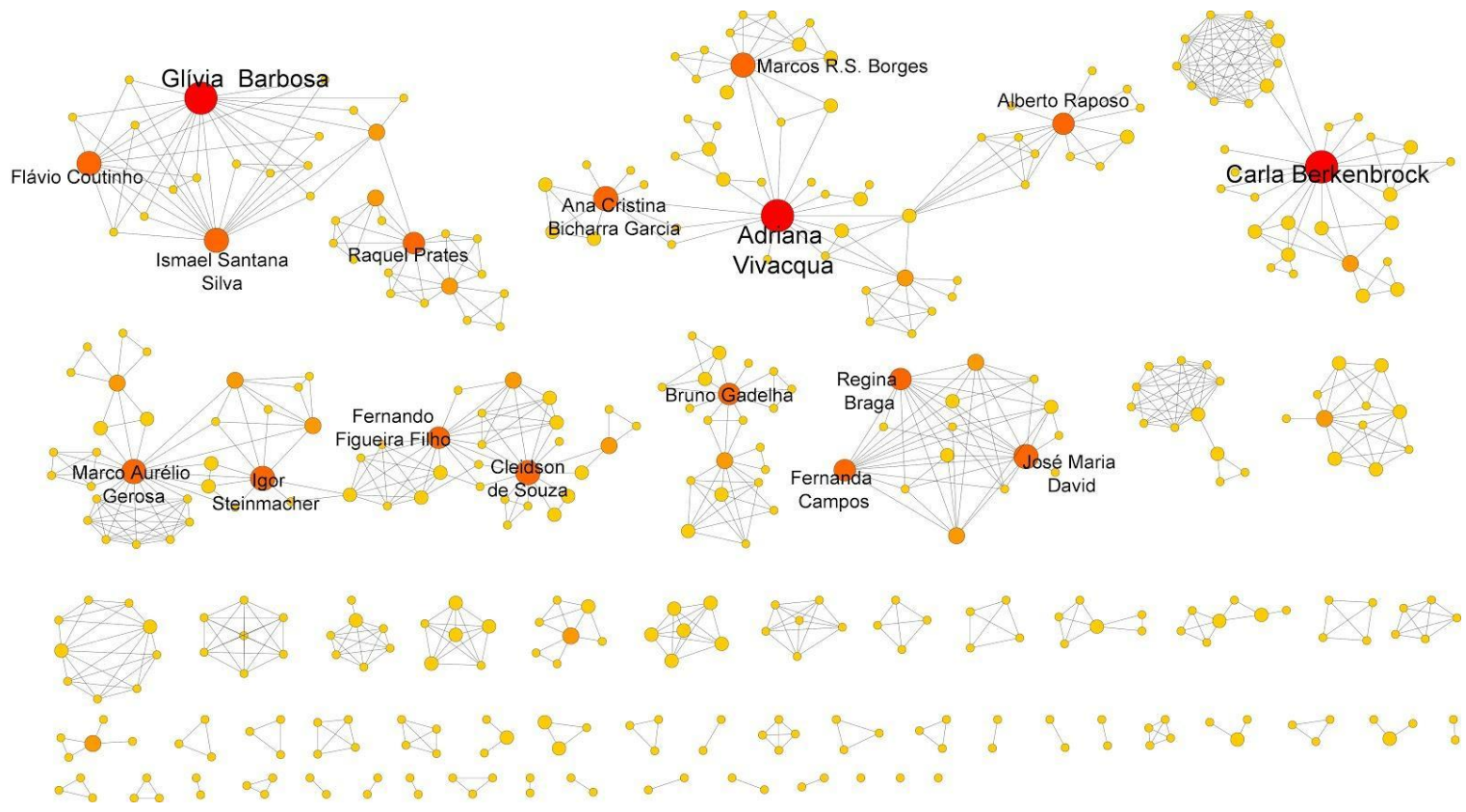

Figura 5. Rede de colaboração científica (2013 a 2019)

Uma análise global dos resultados possibilitou identificar os pesquisadores que mais publicaram nas edições 2013-2019 do SBSC e que, provavelmente, lideram grupos de pesquisa, bem como a formação de novos grupos de colaboração (grupo do Prof. Bruno Gadelha) e expansão de grupos já existentes (grupo do Prof. Cleidson de Souza). Também é possível observar que, dos 411 autores, apenas 105 publicaram mais de um artigo no SBSC e apenas 71 participaram de mais de uma edição do SBSC, mostrando que, apesar do alto número de autores, há pouca constância com o passar dos anos. Além disso, observa-se que a maior parte dos pesquisadores estão divididos em grupos, justificado, muitas vezes, pela relação acadêmica de orientação. Ou seja, apesar da maioria dos autores publicarem em conjunto, existem grupos isolados no SBSC, com poucas colaborações com o restante da rede, inclusive entre autores com os maiores números de publicações (10 ou mais).

Por fim, ao comparar a rede de coautoria mais recente com a rede até 2012 [Steinmacher et al. 2013], observou-se uma mudança nos grupos de pesquisa e autores em destaque, incluindo a formação de novos grupos. Além disso, nota-se uma redução de publicação por parte de alguns autores de destaque até 2012, bem como a formação de grupos fechados de colaboração com pouca interação com demais autores na rede de coautoria mais recente. Também cabe ressaltar que, apesar da rede atual acumulada ser grande, percebe-se que há pouca constância de autores presentes no evento, sendo importante pesquisar o motivo desse acontecimento e evitar novo esvaziamento da comunidade e declínio do evento, como ocorrido antes de 2019.

\section{Conclusões}

Este artigo apresentou uma investigação sobre as redes de colaboração científica no SBSC, entre 2013 e 2019. Os resultados apresentados permitiram identificar certas características da comunidade do SBSC e do evento atualmente. A realização de estudos em torno das redes de eventos científicos auxilia a entender a situação que as pesquisas 
da área se encontram, permitindo também a adaptação e remodelagem dos eventos de modo que continuem relevantes e atualizados, acompanhando a sociedade e suas particularidades, e sempre colocando em pauta discussões atuais.

Este trabalho teve como limitação a análise apenas da comunidade do SBSC, através da documentação do evento disponível na Internet. Nesse contexto, se faz necessário o cadastro de todos os artigos numa plataforma integradora, como a SOL, para fins de consulta e evitar a perda de arquivos importantes do evento, visto que nem todos os pesquisadores publicam seus artigos por conta própria em outras plataformas da Internet. Esta comunidade também está presente em outros eventos científicos nacionais, como o Simpósio Brasileiro sobre Fatores Humanos em Sistemas Computacionais (IHC).

Como próximos passos, está prevista a discussão dos dados levantados junto ao corpo de pesquisadores da comunidade de sistemas colaborativos, visando discutir iniciativas que mitiguem os problemas levantados e potencializem os aspectos positivos encontrados. Além disso, a investigação das questões discutidas neste artigo, considerando outros eventos científicos nacionais que trazem contribuições para a área de sistemas colaborativos.

\section{Referências}

Dias, V.M., Araujo, H., Rodrigues, J. (2014) Sobre as Redes de Coautoria através das edições do SBSC. In Proceedings of the XI Brazilian Symposium in Collaborative Systems

CESC (2017) Assembleia da Comunidade de Sistemas Colaborativos - 05/07/2017. Disponível em: <https://cutt.ly/ElMasR3>

Gasparini, I., Cunha, L.F., Kimura, M.H., Marcelo S.P. (2014) "Análise das redes de coautoria do simpósio brasileiro sobre fatores humanos em sistemas computacionais". In Proceedings of the 13th Brazilian Symposium on Human Factors in Computing Systems (IHC '14). Sociedade Brasileira de Computação, BRA, 323-332.

SBSC (Simpósio Brasileiro de Sistemas Colaborativos) (2019). Disponível em: $<$ https://sites.google.com/ppgi.ufrj.br/sbsc-2019>

Steinmacher, I., Silva, M. A. G., Côgo, F. R., Chaves, A. P., \& Gerosa, M. A. (2013, October). Tópicos de pesquisa e rede de coautoria no Simpósio Brasileiro de Sistemas Colaborativos. In Proceedings of the $X$ Brazilian Symposium in Collaborative Systems (pp. 88-95). 